Draft Version OCtober 24, 2018

Preprint typeset using $\mathrm{L}^{A} \mathrm{~T}_{\mathrm{E}} \mathrm{X}$ style emulateapj v. 08/22/09

\title{
MAGNETIC FIELD EVOLUTION IN RELATIVISTIC UNMAGNETIZED COLLISIONLESS SHOCKS
}

\author{
Uri Keshet $^{145}$, Boaz Katz ${ }^{2}$, Anatoly Spitkovsky ${ }^{3}$, Eli Waxman ${ }^{2}$ \\ (Dated: October 24, 2018) \\ Draft version October 24, 2018
}

\begin{abstract}
We study relativistic unmagnetized collisionless shocks using unprecedentedly large particle-in-cell simulations of two-dimensional pair plasma. High energy particles accelerated by the shock are found to drive magnetic field evolution on a timescale $\gtrsim 10^{4}$ plasma times. Progressively stronger magnetic fields are generated on larger scales in a growing region around the shock. Shock-generated magnetic fields and accelerated particles carry $\gtrsim 1 \%$ and $\gtrsim 10 \%$ of the downstream energy flux, respectively. Our results suggest limits on the magnetization of relativistic astrophysical flows.
\end{abstract}

Subject headings: shock waves - magnetic fields - acceleration of particles — gamma rays: bursts

Due to the low plasma densities, shock waves observed in a wide range of astronomical systems are collisionless, i.e., mediated by collective plasma instabilities rather than by binary particle collisions. Such shocks play a central role in, for example, supernova remnants (Blandford and Eichler 1987), jets of radio galaxies (Maraschi 2003), $\gamma$-ray bursts (GRBs, Piran 2005), pulsar wind nebulae (PWN, Kirk et al. 2007), and the formation of large-scale structure in the Universe (Loeb and Waxman 2000). It is widely accepted that particles accelerated to high energy in such shocks generate the nonthermal radiation observed in a wide range of astrophysical sources and constitute the observed population of cosmic rays.

Despite intense research, collisionless shocks are still not understood from first principles. In particular, there is no self-consistent theory describing the acceleration of particles and the generation of magnetic field fluctuations, which in turn scatter particles and mediate their acceleration. Much of the research has focused on "magnetized" shocks, where the upstream magnetic energy flux constitutes a significant fraction of the total energy flux. Here we focus on "unmagnetized" shocks, where the upstream magnetic energy flux is small. An extreme example of such shocks are the relativistic GRB afterglow shocks, where the magnetic fraction of the energy flux, $\epsilon_{B}$, increases from $\sim 10^{-10}$ in the upstream to $\epsilon_{B} \simeq 0.01-0.1$ in the downstream (Waxman 2006, and references therein). Under such conditions, it is likely that the initial upstream magnetic field does not play a role in the determination of the shock structure (e.g., Gruzinov 2001).

A near equipartition field, $\epsilon_{B} \simeq 0.1$, may be produced by electromagnetic (e.g., Weibel) instabilities (e.g., Blandford and Eichler 1987; Gruzinov and Waxman 1999; Medvedev and Loeb 1999). The coherence length of the generated field is expected to be comparable in

\footnotetext{
${ }^{1}$ Institute for Advanced Study, Einstein Drive, Princeton, NJ, 08540, USA

${ }^{4}$ Benoziyo Center for Astrophysics, Weizmann Institute, Rehovot 7600 , Israel

${ }^{5}$ Department of Astrophysical Sciences, Princeton University, Princeton, NJ 08544, USA

${ }^{4}$ Current address: Center for Astrophysics, 60 Garden St., Cambridge, MA 02138, USA

5 Einstein fellow
}

this case to the plasma skin-depth, $l_{s d}=c / \omega_{p}$, where $\omega_{p}$ is the plasma frequency and $c$ is the speed of light. The main challenge associated with the downstream magnetic field is that while magnetic power on $l_{s d}$ scales rapidly decays after the shock, observations imply that near equipartition fields must persist over $10^{10} l_{s d}$ downstream (Gruzinov and Waxman 1999; Gruzinov 2001). This suggests that the magnetic field develops similarly large coherence lengths (Gruzinov and Waxman 1999; Gruzinov 2001). Such evolution could be driven by large scale currents carried by particles accelerated in the shock, possibly leading to a self-similar plasma configuration (Katz et al. 2007). The mechanism for the generation of large-scale magnetic fields in shocks remains unknown.

Particle-in-cell (PIC) simulations, in which the plasma is represented by macroparticles and Maxwell's equations are solved on a grid, have been extensively used in recent years to study shocks. Such studies have quantified the generation of upstream current filaments by pinching instabilities (e.g., Silva et al. 2003; Frederiksen et al. 2004; Jaroschek et al. 2005; Spitkovsky 2005, 2008a; (Chang et al. 2008), and resolved the formation of shocks in two- and three-dimensional (2D and 3D) pair plasma (Spitkovsky 2005; Kato 2007; Chang et al. 2008) and in 2D ion-electron plasma (Spitkovsky 2008a). These simulations revealed rapid decay of magnetic fields downstream (Gruzinov 2001; Chang et al. 2008), leaving the question of field survival over scales $\gg$ $l_{s d}$ open and triggering alternative suggestions for field generation (e.g., Goodman and MacFadven 2007; Milosavlievic et al. 2007; Sironi and Goodman 2007).

In this Letter, we report new PIC shock simulations performed on unprecedentedly long length and time scales, $\left(L / l_{s d}\right)^{2}\left(T \omega_{p}\right) \simeq 4 \times 10^{10}$. These simulations show the growth of magnetic power on progressively longer scales driven by the accelerated particles, and impose lower limits on the efficiencies of particle acceleration and magnetization. Our results suggest that even the most extensive simulations previously reported (Chang et al. 2008) were too small to capture significant particle acceleration and the resulting magnetic field evolution (see Katz et al. 2007, for a discussion of PIC simulation results and limitations). Here we discuss only the main properties of shock evolution; the particle acceleration 
mechanism is discussed separately (Spitkovsky 2008b), and a detailed analysis of the simulations is deferred to a later publication.

Simulation set-up. For simplicity, we focus here on strong, relativistic shocks in unmagnetized pair plasma. In order to reach long length and time scales, we resort to $2 \mathrm{D}$, and comment below on expected differences with respect to 3D shocks. We use the electromagnetic PIC code TRISTAN-MP (Spitkovskv 2005), a parallel version of TRISTAN (Buneman 1993) heavily modified to minimize noise and numerical instabilities. A rectangular simulation box is set up in the $x-y$ plane, with periodic boundary conditions in the $y$-direction and a conducting wall at $x_{\text {wall }}=0$. Cold, neutral plasma is continuously injected from $x_{i n j}=c t$ in the $-\hat{\mathbf{x}}$ direction, where $t$ is the simulation time. Reflection off the wall then results in a shock propagating along $+\hat{\mathbf{x}}$. All parameters are measured in the downstream frame, in which the wall is at rest.

Typical simulation parameters are: injected bulk Lorentz factor $\gamma_{0}=15$, thermal spread $\Delta \gamma_{0}=10^{-4}$, and $N_{p p c}=8$ particles per species per cell, with spatial and temporal resolutions $\delta x=l_{s d} / 10$ and $\delta t=0.045 \omega_{p}^{-1}$. Here $\omega_{p}^{2}=4 \pi\left(n_{e^{+}}+n_{e^{-}}\right) q^{2} / \gamma_{0} m$, where $m$ and $q$ are the particle mass and charge, and $n$ is the upstream number density. Our largest simulation has $\sim 2 \times 10^{10}$ particles and $\left(L_{x} / l_{s d}\right) \times\left(L_{y} / l_{s d}\right) \times\left(T \omega_{p}\right)=6300 \times 1024 \times 6300 \simeq$ $4 \times 10^{10}$, although smaller simulation boxes have been evolved for as long as $12600 \omega_{p}^{-1}$. The results displayed below mostly refer to a simulation with $L_{y}=402 l_{s d}$, evolved for $T=12600 \omega_{p}^{-1}$.

Short time evolution. At early times, $t \lesssim 1000 \omega_{p}^{-1}$, we recover shock formation as reported previously (Spitkovsky 2005; Chang et al. 2008; Spitkovsky 2008a): a transition layer of a few $10 l_{s d}$ thickness propagating upstream, in which the plasma isotropizes, thermalizes and compresses. The simulated shock transition agrees to within a few percent with (magnetic free) hydrodynamic jump conditions: a shock velocity $v_{s h}=c\left(\Gamma_{d}-1\right)\left[\left(\gamma_{0}-1\right) /\left(\gamma_{0}+1\right)\right]^{1 / 2}$ and density compression ratio $n_{d} / n_{u}=\left(\Gamma_{d}+\gamma_{0}^{-1}\right) /\left(\Gamma_{d}-1\right)$. Here, $\Gamma_{d} \simeq 3 / 2$ is the downstream adiabatic index, and upstream pressure was neglected (Spitkovsky 2008a).

Upstream, the interaction between the unshocked flow and a counterstream running ahead of the shock leads to the formation of current filaments (in both 2D and 3D) parallel to the flow, surrounded by near-equipartition filamentary magnetic (in the fluid frame) structures. Behind the shock, near equipartition magnetic clumps form and are advected with the downstream flow in 2D. At early times (where 3D simulations are possible, $t \lesssim 10^{3} \omega_{p}^{-1}$ ), good agreement is found between these clumps and the $2 \mathrm{D}$ projection of extended magnetic loops formed nearly perpendicular to the flow in $3 \mathrm{D}$ shocks. When averaged along the transverse direction, $\epsilon_{B} \equiv\left(B^{2} / 8 \pi\right) /\left[\left(\gamma_{0}-1\right) n m c^{2}\right]$ (where $B$ is the magnetic field amplitude) peaks at $\sim 7 \%$ near the shock transition layer and decays below $0.1 \%$ within $1000 l_{s d}$ downstream (Chang et al. 2008).

Long time evolution. The above description does not include the effects of high energy particles accelerated by the shock, negligible at early times. Our present simula- tions are sufficiently large to reveal the onset of particle acceleration and the slow evolution of shock properties (evident on $\sim 1000 \omega_{p}^{-1}$ timescales) driven by these energetic particles. A small fraction of particles, accelerated to Lorentz factors $\gamma_{0} \ll \gamma \ll \gamma_{\max }$ by repeated scatterings near the shock, gradually builds up a flat $\left(\gamma^{2} d n / d \gamma \sim\right.$ const.) power-law energy tail downstream, already containing a fraction $\epsilon_{a c c} \gtrsim 10 \%$ of the energy at $t=10^{4} \omega_{p}^{-1}$ (Spitkovskv 2008b). Here we defined $\epsilon_{a c c}$ as the ratio between the energy density of particles with $\gamma>5 \gamma_{0}$ behind the shock, and the far upstream kinetic energy density, such that for a thermal distribution of the particles in the downstream $\epsilon_{a c c}\left(\gamma_{0} \gg 1\right) \simeq 0.3 \%$.
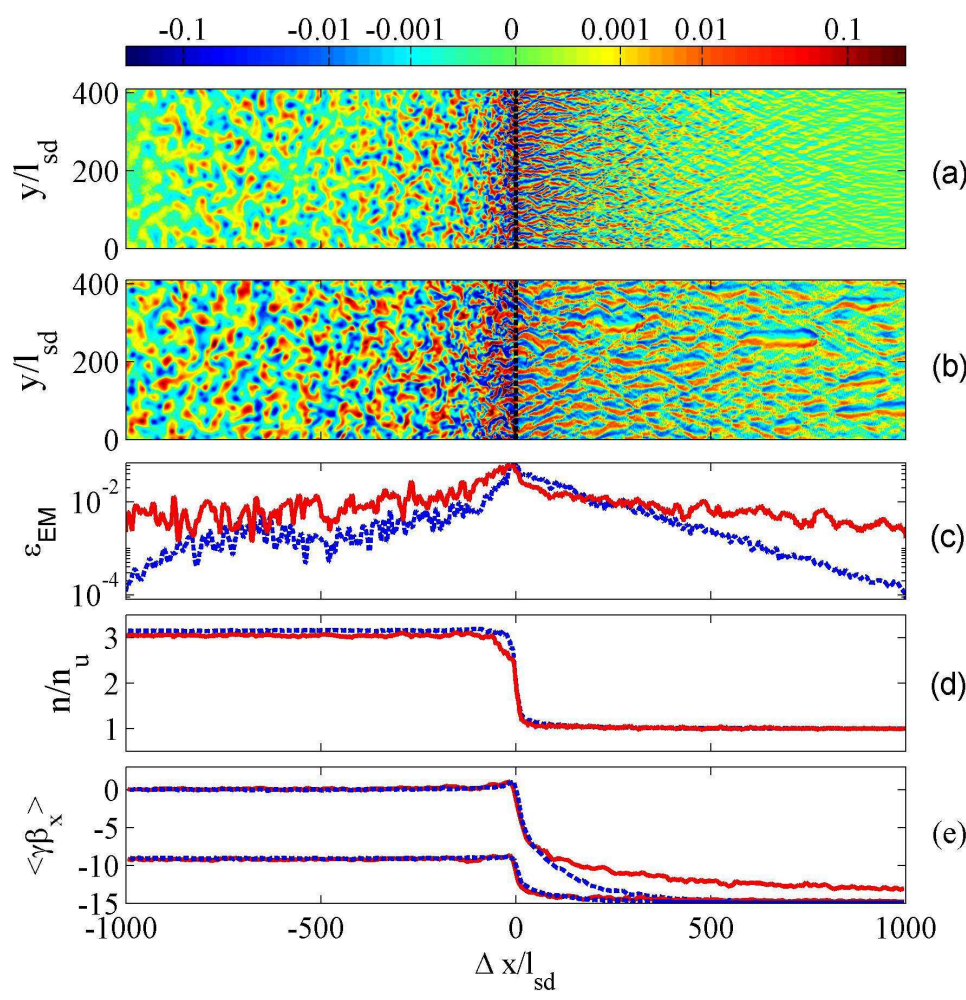

Fig. 1.- Plasma evolution within $1000 l_{s d}$ of the shock. Normalized transverse magnetic field $\operatorname{sign}(B) \epsilon_{B}$ (color scale stretched in proportion to $\epsilon_{B}^{1 / 4}$ to highlight weak features) is shown at (a) early $\left(t_{1}=2250 \omega_{p}^{-1}\right)$, and (b) late $\left(t_{2}=11925 \omega_{p}^{-1}\right)$ times. Here $\Delta x \equiv x-x_{s h}$ is the distance from the shock, with $x_{s h}$ (dashed) defined as median density between far upstream and far downstream. Also shown are the transverse averages (at $t_{1}$, dashed blue, and $t_{2}$, solid red) of (c) electromagnetic energy normalized to the upstream kinetic energy, $\epsilon_{E M} \equiv\left[\left(B^{2}+E^{2}\right) / 8 \pi\right] /\left[\left(\gamma_{0}-1\right) n m c^{2}\right]$ (with $E$ the electric field amplitude, included because in the simulation frame the induced $E \sim B$ upstream), (d) density normalized to the far upstream, and (e) particle momentum $\gamma \beta_{x}$ (with $\beta$ the velocity in $c$ units) in the $\mathrm{x}$-direction averaged over all particles (higher $\left\langle\gamma \beta_{x}\right\rangle$ ) and over downstream-headed particles only.

The energetic particles running ahead of the shock significantly alter the properties of the counterstream and the resulting current filamentation and magnetization upstream. Figure 1 shows the resulting spatial distribution of magnetic fields at early vs. late times, as well as the density and momentum profiles. It reveals an increasing magnetization level, with fields generated on gradually larger scales and extending farther away from the shock, both upstream and downstream. As a result, 
the shock compression transition layer (defined, say, between $10 \%$ and $90 \%$ of full shock compression) widens, $n$ and $\epsilon_{B}$ become more oscillatory with distance behind the shock, the shock slightly accelerates (by $\lesssim 1 \%$ ), and the final compression ratio slightly decreases (by $\lesssim 4 \%$ ). Due to the substantial energy carried by the accelerated particles running ahead of the shock, the average momentum is strongly modified far upstream, $\Delta x \equiv x-x_{s h} \gtrsim 1000 l_{s d}$, although the incoming flow slows down considerably only at $\Delta x \lesssim 100 l_{s d}$ (figure 1e).

Figure 2 quantifies the evolution of downstream magnetization. It shows the power spectrum $P_{k}$ defined by $\epsilon_{B}=\int P_{k} d \log k$ (curves), the average coherence length $\langle\lambda\rangle$ and the energy fraction $\epsilon_{B}$ (filled circles) of magnetic fields in an $l_{x}=800 l_{s d}$ long region trailing behind the shock. In order to avoid shot noise contamination ${ }^{1}, \epsilon_{B}$ is measured only for coherence lengths $\lambda=2 \pi / k>10 l_{s d}$. During $10^{3} \lesssim t \omega_{p} \lesssim 10^{4}, \epsilon_{B}$ in this region grows by a factor of $\sim 4$ and reaches $\sim 1 \%$. As illustrated in Figure 1. the typical size of upstream filaments increases substantially in time, with typical thickness $(10-15) l_{s d}$ at $t=10^{3} \omega_{p}^{-1}$ growing by a factor of $3-4$ by $t=10^{4} \omega_{p}^{-1}$. Figure $[2$ shows a modest effect downstream, where the average magnetic coherence length grows by $\sim 10 \%$ during this epoch.

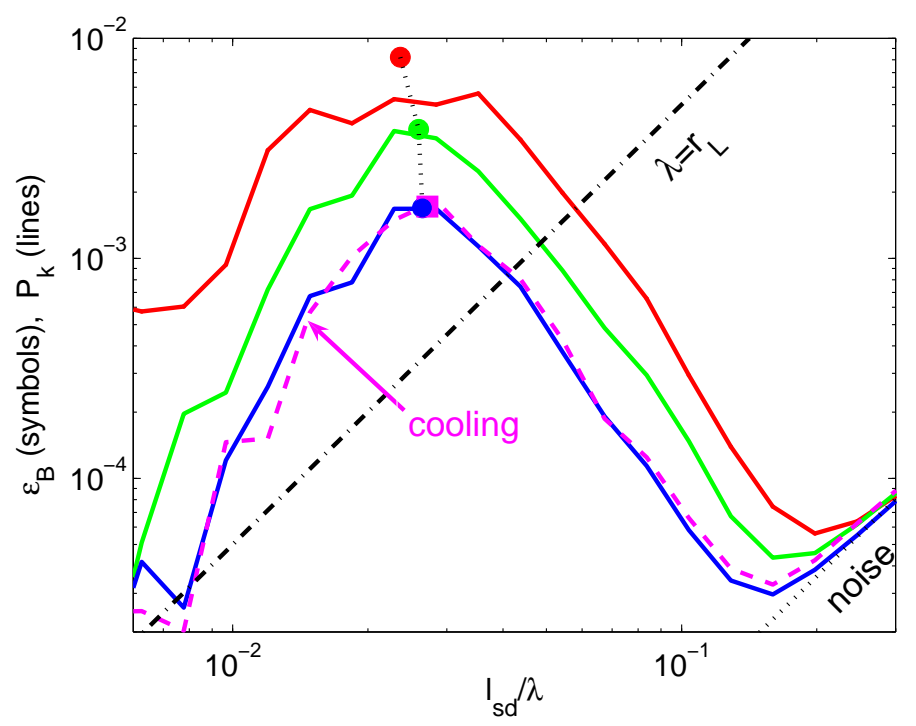

FIG. 2.- Magnetic 2D power spectrum in a sample downstream region defined by $-1000<\Delta x / l_{s d}<-200$. The spectrum is shown to gradually grow and possibly flatten with time [solid curves for $t \omega_{p} \simeq 1900$ (blue), 4600 (green), and 12600 (red)]. The integrated energy fraction $\epsilon_{B}$ and average scale $\langle\lambda\rangle \equiv$ $2 \pi / \exp \left[\epsilon_{B}^{-1} \int \log (k) P_{k} d \log k\right]$ of the magnetic field (filled circles) grow correspondingly. Suppressing particle acceleration (cooling all particles above $\gamma_{c o o l}=80$, dashed line and square for $t \omega_{p}=5750$ ) stops the magnetic evolution. Also shown are estimated shot-noise power (dotted) and a $\lambda=r_{L} \simeq\left(2 P_{k}\right)^{-1 / 2} l_{s d}$ curve (dash dotted) with $r_{L}$ the Larmor radius of $\gamma=\gamma_{0}$ particles, roughly separating magnetized (large scale) and non-magnetized (small scale) bulk plasma regimes.

In order to test the role played by high energy particles in the evolution of the shock, we have performed a

1 Noise with power inversely proportional to $N_{p p c}$, filtered on small scales, and tested not to distort our results. suite of simulations with artificially suppressed particle acceleration. In these runs, we introduced cooling, where particles with $\gamma>\gamma_{\text {cool }}$ lose a random fraction of their nonthermal energy, with various choices of $\gamma_{\text {cool }}$. Cooling is found to significantly slow down or completely stop shock evolution, leading to a fixed magnetization level and a steady-state magnetic power spectrum. Higher values of $\gamma_{\text {cool }}$ are found to produce larger $\epsilon_{B}$ and $\langle\lambda\rangle$. For example, a steady state configuration with $\epsilon_{B}=0.2 \%$ (at $-1000<\Delta x / l_{s d}<-200$ ), obtained for $\gamma_{\text {cool }}=80$ $\left(\gamma_{0}=15\right)$, is shown as a dashed line in Figure 2.

The decay rate of magnetic fields advected downstream slows down as the shock evolves, as illustrated in Figure 3. This is partly attributed to the increased fraction of power deposited in large scale fields, which are expected to survive farther downstream. Indeed, the inset of Figure 3 shows that for $\lambda \gtrsim 40 l_{s d}$, magnetic evolution is well fitted by exponential decay, $P_{k} \propto e^{-\omega t}$, with $\omega \propto k^{2}$. Such behavior is expected, for example, in MHD magnetic diffusion with scale-independent resistivity (note that Figure 2 suggests that the bulk plasma is indeed magnetized on these scales). Figure 3 thus indicates, for example, that power on $\lambda=30 l_{s d}\left(100 l_{s d}\right)$ scales survives more than $10^{3} l_{s d}\left(10^{4} l_{s d}\right)$ downstream.

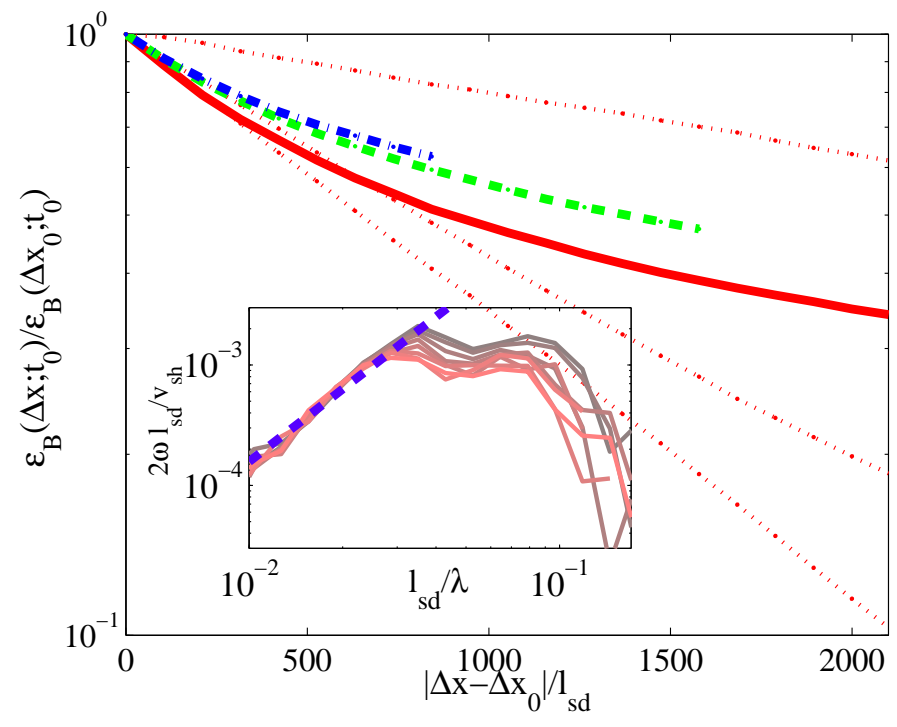

FIG. 3.- Decay of magnetic energy in three different slices advected downstream at progressively later times. Each slice is $l_{x}=$ $1000 l_{s d}$ long, located with its upstream edge at $\Delta x_{0}=-500 l_{s d}$ at $t_{0} \omega_{p}=3600$ (solid red), 5175 (dashed green) and 6750 (dashdotted blue); all curves cutoff when the simulation terminates at $t \omega_{p}=8550$. The decay rate is seen to slow down as the shock evolves. Power on scales $\lambda \gtrsim 40 l_{s d}$ decays nearly exponentially in $|\Delta x|$, shown (dotted red) for $t_{0} \omega_{p}=3600$ and $\lambda / l_{s d}=40,50$ and 67 (fast to slow decay). In Chang et al. (2008), magnetic decay was studied in a fixed downstream frame region (e.g., solid line) not taking into account magnetic field evolution due to accelerated particles.

Inset: Spatial decay rate of the magnetic power spectrum $\left(2 / v_{s h}\right) \omega(k)=P_{k}^{-1}\left(-d P_{k} / d \Delta x\right)$ in a slice (with $l_{x}=1400 l_{s d}$ and $\left.t_{0}=4500 \omega_{p}^{-1}\right)$ advected downstream at $\left(t-t_{0}\right) \omega_{p}=$ $0,225,450, \ldots 1800$ (solid, dark to light curves). At large scales, the decay rate is well fitted by $\omega \propto k^{2}$ (dashed), whereas at small scales $\omega$ decreases with time/distance from the shock.

Convergence. Convergence tests were performed with respect to all simulation parameters (around their val- 
ues given above), with no qualitative changes to the results. Figures 11 and 2 indicate that the simulation box used is sufficiently large to avoid significant boundary effects. However, as 40\% (5\%) of the magnetic power is already deposited in $\lambda>50 l_{s d}\left(\lambda>100 l_{s d}\right)$ scales at $t \simeq 10^{4} \omega_{p}^{-1}$, increasingly larger simulation boxes, in both longitudinal and transverse dimensions, will be required in order to properly resolve the shock and the growing coherent structures.

Conclusions. Our analysis shows that collisionless shock configurations simulated previously may represent steady state configurations only as long as particle acceleration remains insignificant. We find that a population of energetic particles is accelerated and drives the generation of progressively stronger fields on gradually larger scales. Our simulations do not reach a steady state; rather, an increasing fraction of shock energy is transferred to energetic particles and magnetic fields throughout the simulation time domain.

Once stochastic acceleration and magnetization ensue, they are unlikely to diminish to a lower energy steady state. Hence, our results suggest lower limits to the efficiencies of magnetization and particle acceleration, $\epsilon_{B} \gtrsim 1 \%$ at distances $|\Delta x|<D=1000 l_{s d}$ downstream of the shock, and $\epsilon_{a c c} \gtrsim 10 \%$ with no significant cooling identified downstream. We find no evidence for the saturation of $\epsilon_{a c c}, \epsilon_{B}, D$ or $\gamma_{\max }$, although the high energy particles downstream are already sub-equipartition at $t \simeq 10^{4} \omega_{p}^{-1}$.

Although our results are obtained for 2D pair plasma, we expect qualitatively similar shock evolution in 3D shocks and in electron-ion plasma. While the nature of upstream current filaments and downstream magnetic loops/clumps may depend on dimensionality, 2D and $3 \mathrm{D}$ simulations are in good agreement at early times (Spitkovsky \& Arons, in preparation). Also, shocks in ion-electron plasma were found to be similar to pair plasma shocks at early-time $2 \mathrm{D}$ simulations due to effi- cient electron heating (Spitkovsky 2008a). Some level of stochastic particle acceleration is inevitable in all cases, but the generalization of our results to $3 \mathrm{D}$ or to ionelectron plasma is yet to be tested at late times and in the presence of a high energy particle tail.

The major role played by high energy particles in shock evolution, their flat spectrum and the apparently flattening magnetic power spectrum are trends consistent with a self-similar plasma configuration (Katz et al. 2007), although the simulated downstream scale growth is more modest than the $\lambda \propto D$ self-similar scaling. At this stage, the simulations are not yet sufficiently advanced to validate or rule out self-similarity.

In summary, we have shown that collisionless shocks in 2D pair plasma evolve on long, $\gtrsim 10^{3} \omega_{p}^{-1}$ timescales, such that the acceleration efficiency, magnetization level, and coherence length scale all increase in time. These trends and the above lower limits on $\epsilon_{a c c}$ and $\epsilon_{B}$ indicate that a shock propagating into a cold, homogeneous plasma with $B=0$ remains a viable model for astronomical shocks, with no need for additional assumptions about magnetic turbulence generation (e.g., Goodman and MacFadven 2007; Milosavlievic et al. 2007; Sironi and Goodman 2007). Our results confirm that particle acceleration and magnetization are intimately related, with high energy particles playing a major role in generating the magnetic fields which in turn scatter and accelerate the particles.

We thank J. Arons and P. Goldreich for helpful discussions. U.K. is a Friends of the Institute for Advanced Study member, and is supported by NSF grant PHY0503584. The research of B. K. \& E. W. is partially supported by AEC, ISF \& Minerva grants. A.S. acknowledges the use of computational resources at TIGRESS computing center at Princeton University and the support from Alfred P. Sloan Foundation fellowship.

\section{REFERENCES}

R. Blandford and D. Eichler, Phys. Rep. 154, 1 (1987).

O. Buneman, in Computer Space Plasma Physics, edited by H. Matsumoto and Y. Omura (Terra Scientific, Tokyo, 1993), p. 67.

P. Chang, A. Spitkovsky, and J. Arons, ApJ 674, 378 (2008).

J. T. Frederiksen, C. B. Hededal, T. Haugbølle, and Å. Nordlund, ApJ 608, L13 (2004).

J. Goodman and A. MacFadyen, ArXiv e-prints (2007), arXiv:0706.1818.

A. Gruzinov, ApJ 563, L15 (2001).

A. Gruzinov and E. Waxman, ApJ 511, 852 (1999).

C. H. Jaroschek, H. Lesch, and R. A. Treumann, ApJ 618, 822 (2005).

T. N. Kato, ApJ 668, 974 (2007).

B. Katz, U. Keshet, and E. Waxman, ApJ 655, 375 (2007).

J. G. Kirk, Y. Lyubarsky, and J. Petri (2007), arXiv:astro-ph/0703116.

A. Loeb and E. Waxman, Nature 405, 156 (2000)

L. Maraschi, in Active Galactic Nuclei: From Central Engine to Host Galaxy, edited by S. Collin, F. Combes, and I. Shlosman (2003), vol. 290 of Astronomical Society of the Pacific Conference Series, p. 275.
M. V. Medvedev and A. Loeb, ApJ 526, 697 (1999).

M. Milosavljevic, E. Nakar, and F. Zhang, ArXiv e-prints (2007), arXiv:0708.1588.

T. Piran, Reviews of Modern Physics 76, 1143 (2005).

L. O. Silva, R. A. Fonseca, J. W. Tonge, J. M. Dawson, W. B. Mori, and M. V. Medvedev, ApJ 596, L121 (2003).

L. Sironi and J. Goodman, ApJ 671, 1858 (2007).

A. Spitkovsky, in Astrophysical Sources of High Energy Particles and Radiation, edited by T. Bulik, B. Rudak, and G. Madejski (2005), vol. 801 of American Institute of Physics Conference Series, pp. 345-350.

A. Spitkovsky, ApJ 673, L39 (2008a).

A. Spitkovsky, ApJ 682, L5 (2008b).

E. Waxman, Plasma Physics and Controlled Fusion 48, B137 (2006). 